\title{
Uso delle medicine complementari in nefrologia: una revisione di studi randomizzati controllati (RTC)
}

\author{
Lisa Bovo ${ }^{1}$, Francesco Burrai ${ }^{2}$, Fabiola Carossa ${ }^{3}$ \\ ${ }^{1}$ Infermiera libero professionista, Padova \\ ${ }^{2}$ Servizio Professioni Sanitarie, ATS Sardegna - ASSL Olbia, Olbia \\ ${ }^{3}$ Oncologia Medica, Istituto Oncologico Veneto IOV-IRCSS, Padova
}

\begin{abstract}
Use of complementary and alternative medicine in nephrology: a review of RCTs
Purpose: To evaluate and summarize the current evidence in the literature on the effectiveness of complementary and alternative medicine (CAM) for the management of health problems in kidney patients.

Methods: Randomized controlled trials in English were selected through PubMed, Medline, the Cochrane Central Register of Controlled Trials, and CINAHL. There were no time limits on the research.

Results: Thirty-four articles were selected for the review. Most of the studies were reported in 2013 and 2014 and were conducted in China. Multiple types of CAM used for various problems in kidney patients (pruritus, reduced respiratory capacity, poor quality of sleep, abnormal blood values, pain, anxiety) emerged from the analyzed studies. The most studied types of CAM were the herbs used in Chinese medicine that can help to control proteinuria, creatinine and hematuria, and to reduce the dose of traditional medicines; acupuncture, which can relieve pruritus and disturbed sleep; respiratory training, which increases the respiratory capacity and muscle strength; and Benson relaxation, which helps to improve pain and sleep.

Conclusions: Although the evidence of the included studies is limited, CAM may be helpful for the treatment and control of health problems in kidney patients. To actually recommend its use, further studies are needed.
\end{abstract}

Keywords: Complementary therapies, Nephrology, Randomized controlled trial

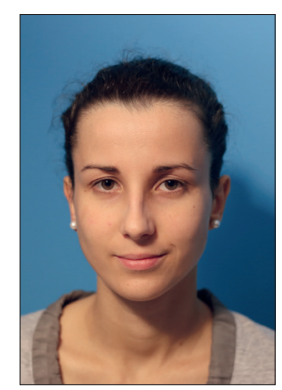

Lisa Bovo

\section{Introduzione}

I pazienti con malattie renali hanno un aumentato rischio di malattia cardiovascolare rispetto alla popolazione generale. Ogni anno circa il $10-20 \%$ dei pazienti in dialisi muore per complicanze cardiovascolari, ipertensione, dislipidemia e squilibrio della ionemia (1). Le attuali opzioni di trattamento includono sia le terapie convenzionali sia le terapie complementari (CAM), che vengono utilizzate per alleviare i sintomi e le complicanze (2). Le CAM ormai riconosciute dal mondo scientifico presentano

Accepted: June 26, 2017

Published online: September 13, 2017

Indirizzo per la corrispondenza:

Dr.ssa Lisa Bovo

Via Cornara, 103

35010 Villanova di Camposampiero (PD)

bovolisa@gmail.com evidenze che si integrano in maniera sinergica con l'assistenza convenzionale. Sebbene le CAM siano conosciute a livello mondiale il loro uso in dialisi è ancora poco praticato e poco conosciuto (3). II paziente nefrologico può accusare molti sintomi quali: prurito, difficoltà respiratoria, dolore e ansia, peggioramento della qualità del sonno, ansia e ipotensione intradialitica (4). Vi è un'aumentata incidenza di diabete, anemia e malattia vascolare periferica, ipertensione, malattie coronariche e infarti. La persona in malattia renale terminale ricorre correntemente a un trattamento emodialitico e la capacità aerobica arriva solo alla metà dei valori normali (4). Varie sono le opzioni di trattamento in associazione alle terapie convenzionali: il rilassamento, l'agopuntura, lo yoga, il training respiratorio, la musicoterapia, la medicina cinese, la fitoterapia (1, 4-16). L'uso delle CAM è ormai diventato una strategia comune secondo molti studi e si è osservato che molti sono i pazienti che utilizzano e credono che le terapie complementari siano sicure rispetto ai farmaci, chiedono consiglio ai loro amici e pochi però chiedono consiglio ai medici (3). Le CAM possono offrire un approccio integrato per la gestione dei problemi nei pazienti nefropatici, per esempio nel controllo del dolore, migliorare la qualità del sonno, migliorare la capacità respiratoria, alleviare il prurito, tenere sotto controllo i valori ematochimci, tenere sotto controllo la 
pressione arteriosa $(7,17-25)$.

I pazienti affetti da malattia renale che si sottopongono a queste procedure o assumono questi trattamenti CAM devono essere monitorati in quanto vi è un aumentato rischio di tossicità dovuto all'assenza sia totale che parziale delle funzioni renali. Gli studi randomizzati controllati (RCT) individuati nei nostri due articoli, hanno come oggetto di applicazione le seguenti variabili dipendenti: prurito, capacità respiratoria, dolore, ansia, qualità del sonno, valori ematochimici.

\section{Materiali e metodi}

\section{Scopo}

Lo scopo di questa revisione è quello di valutare e sintetizzare gli attuali studi RCT sull'efficacia delle CAM in ambito nefrologico.

\section{Obiettivi}

L'obiettivo di questa revisione è quello di valutare se l'utilizzo delle CAM porta benefici al paziente nefrologico e di mostrare che tipo di benefici producono.

\section{Disegno}

È stata condotta una revisione della letteratura di studi randomizzati controllati (RCT) per l'individuazione di possibili effetti della CAM nel trattamento di svariate problematiche presenti nei pazienti nefrologici.

\section{Metodi di Ricerca}

Sono stati consultati quattro database elettronici: PubMed, Medline, The Cochrane Central Register of Controlled Trials, Cinahl.

Le parole chiave inserite sono state:

- "Complementary Therapies" MeSh Term comprendente: Acupuncture Therapy, Acupuncture Analgesia, Acupuncture Ear, Electroacupuncture, Meridians, Moxibustion, Anthroposophy, Auriculotherapy, Diffuse Noxious Inhibitory Control, Holistic Health, Bioresonance Therapy, Homeopathy, Horticultural Therapy, Traditional Medicine, African Traditional Medicine, Arabic Medicine, Ayurvedic Medicine, East Asian Traditional Medicine, Shamanism, Mesotherapy, Mind-Body Therapies, Aromatherapy, Psychology, Breathing Exercises, Hypnosis, Imagery (Psychotherapy), Laughter Therapy, Meditation, Mental Healing, Psychodrama, Psychophysiology, Relaxation Therapy, Taiji (Tai Chi), Therapeutic Touch, Yoga, Musculoskeletal Manipulations, Kinesiology, Chiropractic Manipulation, Osteopathic Manipulation, Soft Tissue Therapy, Naturopathy, Organotherapy, Tissue Therapy Historical, Phytotherapy, Eclecticism,
Reflexotherapy, Sensory Art Therapies, Acoustic Stimulation, Art Therapy, Color Therapy, Dance Therapy, Music Therapy, Play Therapy, Speleotherapy, Spiritual Therapies, Faith Healing, Magic, Meditation, Mental Healing, Radiesthesia, Witchcraft.

- Kidney, Renal, Dialysis, Nephrology.

\section{Criteri di inclusione/esclusione}

Popolazione: nefropatici di tutte le età; Intervento: tutte le "complementary and alternative medicine" incluse nel MeSh Term "Complementary Therapies"; Comparazione: CAM versus nessun intervento, versus medicina classica, versus altra tipologia di CAM; Outcome: tutti gli esiti legati all'utilizzo delle CAM nei pazienti nefropatici; Lingua: solo inglese; Disegno degli studi: RCT. Nella ricerca non sono stati imposti limiti temporali alle date delle pubblicazioni. Gli articoli non disponibili in full text sono stati esclusi.

\section{Risultati}

Nella revisione sono stati inclusi 34 articoli di studi RCT: 7 risalenti agli anni tra il 2003 e il 2005, 6 tra il 2006 e il 2011, 3 articoli sono del 2012, 7 del 2013, 5 del 2014 mentre altri 6 tra il 2015 e il 2016. La maggior parte degli studi è stata condotta in Cina $(n=9)$, in Iran $(n=7)$, in Taiwan $(n=4)$ e in Brasile $(n=4)$. Altri studi sono stati svolti in Turchia, Israele, India, Gran Bretagna e USA. Un solo studio è stato svolto in più Paesi (Italia, Germania, Spagna, Francia). Dei 34 articoli inclusi nella ricerca, 15 RCT studiavano gli effetti delle CAM per migliorare i valori ematochimici, 4 RCT per il miglioramento del prurito, 2 RCT per aumentare la capacità respiratoria, $4 \mathrm{RCT}$ per la riduzione del dolore, $8 \mathrm{RCT}$ per migliorare la qualità del sonno, 1 RCT per migliorare l'ansia.

Le caratteristiche di questi RCT sono sintetizzati nella Tabella Supplementare S1 (disponibile online su www.gtndonline.com).

Di seguito vengono illustrati i risultati mostrati dagli RCT divisi per outcome.

\section{Valori ematochimici}

Tredici studi RCT hanno esaminato l'uso di erbe della medicina tradizionale cinese. Chen et al (19) hanno osservato come la proteinuria si presenti in persone con nefropatia membranosa idiopatica e le cause siano soprattutto legate all'uso di prednisone e ciclofosfamide. In questo studio RCT i pazienti con nefropatia che presentavano proteinuria sono stati divisi in due gruppi. Al gruppo di controllo sono stati somministrati prednisone e ciclofosfamide e l'altro gruppo veniva trattato con l'erba Shenqi particle. Dopo 48 settimane di terapia si è notato un miglioramento $(p<0,001)$ della proteinuria nel gruppo Shenqi particle, mentre nel gruppo di controllo vi sono stati cambiamenti nel livello della Glomerular Filtration Rate (eGFR) ma con effetti più contenuti $(p=0,5)$. 
Nello studio di Li et al (7) sono stati coinvolti 45 pazienti con glomerulonefrite, ematuria e proteinuria, ai quali veniva somministrata l'erba Huai Qi Haung. Dopo otto settimane ( $p=0,007)$ e dopo dodici settimane $(p=0,001)$ l'escrezione di proteine era significativamente ridotta ed era associata a una completa remissione di ematuria $(p=0,001)$ senza eventi avversi. Wang et al (15) hanno utilizzato l'agopuntura auricolare, valutando il miglioramento della qualità di vita nelle persone con malattia cronica renale. I pazienti sono stati divisi in due bracci, sperimentale e di controllo, con misurazioni dell'HbA1c, dell'eGFR e della qualità di vita con il Kidney Disease Quality of Life Short Form (KDQOL-SF) in baseline e dopo tre mesi di trattamento con agopuntura. I risultati hanno mostrato che i pazienti sottoposti ad agopuntura hanno riportato punteggi superiori sul KDQOL-SF rispetto al gruppo di controllo dopo tre mesi di trattamento con agopuntura $(p=0.001)$. Nello studio di Singh et al (11) sono state utilizzate la Salacia oblonga e la Salacia chinensis per studiarne gli effetti sulla progressione della malattia e sul livello di diversi marcatori ematici in comparazione al gruppo di controllo placebo.

I risultati hanno mostrato come la Salacia chinensis può ritardare la progressione della malattia e ridurre la progressione di omocisteina sierica e creatinina $(p=0,05)$, mentre la Salacia chinensis può avere un effetto protettivo $(p=<0,05)$. Singh et al (12) hanno studiato gli effetti della Salacia oblon$g a$ in 60 pazienti divisi in quattro gruppi, con misurazioni dei livelli di diversi marcatori ematici a tre mesi e a sei mesi. La Salacia oblonga riduce i trigliceridi $(p=0,008$ ) nei pazienti non diabetici, mentre la stabilizzazione della clearance della creatinina con Salacia oblonga è stata osservata sia nei pazienti non diabetici $(p=0,05)$ sia nei diabetici $(p=0,04)$. Effetti significativi sono stati mostrati sia sul profilo lipidico ( $p=0,0001)$, che sui marker di infiammazione endoteliale ( $p=0,02)$ e sulla interleukin-6 ( $p=0,0003)$. Nello studio di Zhang et al, l'uso di Abelmoschus manihot ha mostrato una riduzione della proteinuria $(p=0,01)(25)$. Lemos et al (20) hanno sperimentato l'uso di oli di semi di lino in tre unità operative in pazienti emodializzati suddivisi in due gruppi bilanciati: 80 pazienti hanno ricevuto oli minerali e gli altri 80 hanno ricevuto oli di semi di lino. L'uso di oli di semi di lino ha ridotto lo stato infiammatorio correlato a un riduzione del BMI, delle lipoproteine e del colesterolo $(p=0,01)$.

Nello studio di Wu et al (23), pazienti che hanno effettuato trapianto renale con effetti collaterali da ciclosporina, sono stati trattati con Berberina. I valori epatici e la concentrazione di Cyclosporine (CsA) ematica hanno mostrato un trend positivo verso i livelli normali $(p=0,05)$ rispetto ai pazienti che sono stati trattati con placebo. Un'altra pianta della medicina tradizionale cinese la Zishen Tongli Jiaonang è stata utilizzata per le infezioni del tratto urinario, mostrando un tasso di infezione del $4,4 \%$ nel gruppo di trattamento e del $30 \%$ in quello di controllo $(p<0,01)$ $(16,24)$. Nello studio di Qui et al (9) è stato utilizzato general acetoside di Rehmannia e piperazine ferulate. Un campione di 400 pazienti con diagnosi di glomerulonefrite primaria è stato ran- domizzato in due gruppi: un gruppo sperimentale trattato con general acetoside di Rehmannia e un gruppo di controllo trattato con piperazine ferulate. Dopo 8 settimane, nel gruppo trattato con general acetoside di Rehmannia si è osservata una riduzione della proteinuria $(p=0,001)$ e della eritrocituria $(p=0,001)$.

Nello studio di Shema-Didi et al (1), 100 pazienti del gruppo sperimentale sono stati trattati con succo di melograno rispetto al gruppo placebo. II gruppo sperimentale ha mostrato una riduzione della pressione sistolica $(p=<0,001)$, dei trigliceridi $(p=0,02)$, e dei livelli di HDL $(p=0,01)$.

\section{Prurito}

II prurito uremico è un problema comune nei pazienti con ESRD (End Stage Renal Disease) in emodialisi. La sua prevalenza stimata è del 30-50\%. L'eziologia è ancora sconosciuta, ma dagli studi emergono cause multifattoriali: squilibrio del calcio, fosforo e magnesio, aumento di mastociti cutanei e istamina. Le terapie tradizionali si focalizzano nel trattare queste possibili cause $(26,27)$.

Quattro studi hanno mostrato come l'utilizzo di alcuni tipi di CAM possano alleviare il prurito uremico in pazienti dializzati. Cavalcanti et al (18) hanno utilizzato dei prodotti omeopatici in un gruppo di 11 pazienti brasiliani in dialisi, che soffrivano di un moderato o severo prurito senza cause dermatologiche. Il più prescritto è stato Calcarea phosphorica. Nel gruppo trattato con l'omeopatia, l'intensità del prurito diminuisce significativamente rispetto ai valori iniziali $(p=0,003)$. In un altro studio, è stata utilizzata una lozione a base di Avena sativa spalmata due volte al giorno per due settimane nelle zone dove i pazienti in dialisi lamentavano prurito. I risultati non hanno dimostrato differenze significative $(p=0,15)$ tra il gruppo sperimentale e i due gruppi controllo ai quali veniva somministrata una lozione a base di aceto oppure un antistaminico (26).

Un'altra tipologia di CAM per alleviare il prurito è stata l'agopuntura. In uno studio, l'agopressione è stata praticata per un'ora, tre volte a settimana, per un mese, in due gruppi di 20 pazienti in dialisi: in un gruppo l'ago è stato applicato nel punto Quchi (LI11) (all'estremità laterale della piega del gomito) mentre nel secondo gruppo l'ago è stato applicato a 2 centimetri di distanza dal punto esatto in cui il paziente lamentava prurito. Dopo un mese di trattamento il primo gruppo ha presentato una diminuzione significativa del prurito rispetto al secondo gruppo $(p<0,001)$, con mantenimento costante anche dopo tre mesi dalla fine del trattamento (27).

Nella ricerca di Hsu et al (2) è stata utilizzata una terapia termale (Bio Worm) con raggi infrarossi diretti nel punto Sanyinjiao, per 15 minuti al giorno, per 18 sedute, in 21 pazienti in emodialisi. II prurito è stato misurato attraverso una forma short del McGill Pain Questionnaire. Nei pazienti del gruppo sperimentale il prurito è diminuito notevolmente $(p<0,001)$ ma non c'è stata differenza significativa con il gruppo controllo ( $p=0,001)$. 


\section{Capacità respiratoria}

I pazienti con ESRD vengono sottoposti di solito a tre o quattro sedute di emodialisi a settimana di durata ciascuna di circa 3-4 ore. La patologia renale di base e queste lunghe sessioni di dialisi possono predisporre i pazienti a ipotensione, ipossia, crampi, affaticamento muscolare e diminuzione della capacità respiratoria $(6,8)$. Due studi hanno indagato gli effetti sulla capacità respiratoria utilizzando programmi di training respiratorio. Figueiredo et al (6) hanno condotto uno studio RCT con 44 pazienti con ESRD in emodialisi per valutare gli effetti degli esercizi respiratori sul rafforzamento dei muscoli respiratori. Gli esercizi venivano praticati per 20 minuti tra la seconda e la terza ora di emodialisi, tre giorni alla settimana, per sei settimane. Il training respiratorio consisteva in un minuto di respirazione con inspirazione ed espirazione forzata massima, al fine di allungare le fibre dei muscoli respiratori, sviluppando una migliore contrazione muscolare, alternato a un minuto di riposo. Nel gruppo sperimentale c'è stato un miglioramento significativo nei valori della Capacità Vitale Forzata (FVC) $(p=0,001)$, del Volume Espiratorio Massimo nel I Secondo $\left(F_{1} V_{1}\right)(p<0,001)$, della Massima Pressione Inspiratoria (MIP) $(p<0,0001)$ e della Massima Pressione Espiratoria (MEP) $(p=0,002)$ rispetto alla fase baseline e al gruppo di controllo.

Uno studio simile è stato condotto da Pellizzaro et al (8) che hanno coinvolto 45 pazienti in emodialisi con ESRD divisi in tre gruppi: controllo, Respiratory MuscleTraining (RMT) e Peripheral Muscle Training (PMT). Per dieci settimane, durante le prime due ore di dialisi, i pazienti del gruppo RMT eseguivano esercizi per i muscoli inspiratori mentre i pazienti del gruppo PMT con i muscoli estensori delle ginocchia. Nel gruppo RMT la MIP e la MEP sono aumentate notevolmente sia rispetto al gruppo controllo $(p<0,001)$ sia rispetto al gruppo $\operatorname{PMT}(p=0,01)$. Non c'è stata differenza significativa nei valori della FVC $(p=0,58)$.

Nel test di camminata (Six-minute walk test) c'è stato un significativo aumento della distanza percorsa nel gruppo RMT $(p<0,001)$ e nel gruppo PMT $(p=0,019)$ rispetto al gruppo controllo. Infine è stata analizzata anche la qualità di vita con il KDQOL-SF, dove i risultati hanno mostrato un incremento del punteggio nei due gruppi sperimentali sul sonno $(p<0,001)$, sul dolore $(p<0,001)$ e sulla fatigue $(p=0,002)$ rispetto al gruppo controllo.

\section{Dolore}

Uno dei problemi più comuni nei pazienti con ESRD e in emodialisi è il dolore cronico, e più del $50 \%$ dei pazienti lamenta dolore in più sedi. II dolore è in gran parte causato dall'inserimento dell'ago nella fistola ma possono soffrire di dolore osseo da osteodistrofia renale, neuropatia periferica, artropatia dialisi-correlata, cefalea e crampi muscolari durante o dopo l'emodialisi $(17,21)$.

Il dolore è quindi un aspetto critico nella vita di un pa- ziente nefrologico soprattutto durante le sedute di emodialisi. Bagheri-Nesami et al (17) hanno sperimentato l'aromaterapia come possibile rimedio al dolore provocato dall'inserimento dell'ago nella fistola. Lo studio ha coinvolto 92 pazienti con ESRD divisi in due gruppi: al gruppo sperimentale per tre sedute consecutive è stata somministrata, a $10 \mathrm{~cm}$ di distanza dal naso, un'essenza a base di lavanda al $10 \%$, che veniva inspirata lentamente per 5 minuti. Al termine del trattamento, veniva inserito un ago FAV di solito calibro e tipo. II gruppo di controllo invece riceveva un'essenza placebo. Il dolore è stato misurato con la scala VAS e, dopo tre interventi, c'è stata una notevole diminuzione dell'intensità del dolore nel gruppo sperimentale rispetto al gruppo controllo $(p<0,001)$. Uno studio simile (28) è stato effettuato per valutare gli effetti della lavanda sulla fatigue. L'essenza di lavanda al $5 \%$ veniva inalata per 10 minuti, durante la dialisi, per tre volte alla settimana per un mese.

La fatigue, misurata con la Fatigue Severity Scale (FSS), non è diminuita significativamente rispetto al gruppo di controllo $(p>0,05)$.

Ozdemir et al (21) hanno utilizzato la riflessologia in 80 pazienti, la quale veniva praticata durante l'emodialisi, per 30 minuti, nei piedi nei punti corrispondenti alle sedi dove i pazienti accusavano dolore, fatigue e crampi. Dopo la terza seduta di riflessologia, veniva misurato il dolore con la scala VAS, con risultati che mostravano un aumento dei livelli di dolore nel gruppo controllo mentre è diminuito nel gruppo di intervento $(p<0,05)$.

In uno studio è stato utilizzato il rilassamento Benson in 86 pazienti in emodialisi (29). Il gruppo sperimentale praticava la tecnica Benson due volte al giorno per 20 minuti: chiudere gli occhi, rilassare i muscoli a partire dai piedi fino a tutto il corpo e ascoltare attentamente il suono della respirazione. Dopo otto settimane l'intensità del dolore nel gruppo sperimentale è diminuita rispetto al gruppo controllo $(p=0,01)$.

\section{Ansia}

Molti pazienti in emodialisi che affrontano la malattia, mostrano spesso problemi psicologici come la depressione e I'ansia. Solo uno studio ha esaminato l'effetto di una CAM, ovvero la musicoterapia sull'ansia dei pazienti in dialisi. Nello studio di Cantekin e Tan (5), 50 pazienti sono stati sottoposti a musicoterapia (brani di musica d'arte turca) durante tre sedute di dialisi ed erano liberi di ascoltarla anche al di fuori della dialisi, mentre altri 50 pazienti facevano parte del gruppo controllo. L'ansia misurata con la State-Trait Anxiety Inventory (Stai form TX) ha mostrato al termine delle tre sedute una differenza significativa tra i due gruppi $(p<0,05)$.

\section{Qualità del Sonno}

Una percentuale di pazienti in emodialisi compresa tra il 40 e I' $85 \%$ soffre di disturbi del sonno. Questo problema è 
spesso collegato a diversi fattori come: concentrazioni plasmatiche di tossine (creatinina), anemia, apnee notturne che possono provocare sonnolenza e irritabilità diurna, confusione, depressione (10). Molte ricerche sono state condotte sull'uso delle CAM per migliorare il sonno dei pazienti nefrologici. Shariati et al (22) hanno studiato l'agopuntura in 48 pazienti ESRD in dialisi. Al gruppo trattamento, un'ora dopo I'inizio della dialisi per 15 minuti, veniva applicata l'agopuntura nei punti specifici per migliorare il sonno: Shenmen (HE7) e He Gu (LI4) nelle mani e Sanyinjiao (SP6) nel piede. Dopo quattro settimane di trattamento, i risultati hanno mostrato un miglioramento del sonno, misurato con la Pittsburgh Sleep Quality Index (PSQI), nel gruppo sperimentale significativo rispetto al gruppo controllo ( $p<0,001)$. In un altro studio (30), 108 pazienti in emodialisi sono stati suddivisi in tre gruppi. Al gruppo sperimentale veniva praticata l'agopressione con il pollice nei punti Shenmen (HT7) del polso un'ora dopo la dialisi, per tre volte alla settimana, per un mese, nel secondo gruppo veniva praticata a $0,5 \mathrm{~cm}$ a lato dei punti Shenmen e al terzo gruppo non veniva applicato nessun trattamento. Al termine dei trattamenti è stata osservata una differenza significativa nel punteggio totale della PSQI tra il gruppo sperimentale e gli altri due gruppi $(p<0,001)$. Nello studio di Rambod et al (10) è stato utilizzato il rilassamento Benson su un campione di 86 pazienti in emodialisi. I risultati hanno mostrato una differenza significativa tra il gruppo sperimentale e il gruppo controllo nel punteggio totale della PSQI $(p<0,0001)$. Nello studio di Yurtkuran et al (4) un campione di 40 pazienti in emodialisi è stato studiato per esaminare gli effetti dello yoga sul sonno. Il gruppo sperimentale praticava esercizi di base di yoga (ritmo lento e coordinato della respirazione, focalizzarsi sui feedback del corpo) per 30 minuti, due volte al giorno, per tre mesi. La qualità del sonno è migliorata nel gruppo sperimentale $(p=0,04)$. Per migliorare la qualità del sonno è stata utilizzata anche la riflessologia (31), la quale veniva praticata a livello dei piedi, per 30 minuti, due volte alla settimana, per un mese, nei pazienti prima che si sottoponessero alla dialisi. I risultati hanno mostrato un miglioramento della qualità del sonno significativa $(p<0,001)$ sulla scala PSQI e sul livello della fatigue sulla scala Visual Analogue Fatigue Scale (VASF), rispetto al gruppo in cui veniva praticato il massaggio alla schiena e rispetto al gruppo di controllo. Tsay ha condotto diversi studi sulla qualità del sonno. Nel 2003 ha studiato gli effetti dell'agopuntura (14), la quale veniva praticata per 14 minuti, tre volte a settimana, per quattro settimane, nei punti Shenmen (HT7) nelle orecchie e nelle mani e Yungchuan (K11) nei piedi. I risultati sulla scala PSQI mostravano differenze significative tra il gruppo sperimentale e il gruppo controllo sulla qualità percepita del sonno durante la notte $(p=0,002)$, sulla qualità del sonno soggettivo $(p=0,009)$, sulla durata del sonno $(p=0,004)$, sull'efficienza del sonno abituale $(p=0,001)$ e sulla sufficienza del sonno ( $p=0,004)$.

Nel 2004 Tsay ha riproposto lo stesso studio (13), utilizzando l'agopuntura nei punti Yungchuan (K1) nei piedi, Zu- sanli (ST36), Yanglingquan (GB34) e Sanyinjiao (SP6) nelle gambe. II gruppo sperimentale ha mostrato punteggi inferiori rispetto al gruppo controllo ma non significativi $(p>0,05)$ in quanto i punti coinvolti erano specifici per diminuire la fatigue $(p=0,001)$. Per il sonno disturbato è stato studiato anche il training respiratorio. In uno studio (32) su un campione di 57 pazienti in emodialisi, il gruppo sperimentale praticava, durante otto sedute di dialisi, 20 minuti di respirazione guidata. Non ci sono state differenze significative tra i due gruppi per quanto riguarda i punteggi nella PSQI $(p=0,5)$, ma ci sono state differenze significative a livello della depressione misurata con la scala Beck Depression Inventory $(p=0,01)$ e nella qualità di vita misurata con la Medical Outcome Studies 36-Item Short Form Health Survey $(p=0,009)$.

\section{Discussione}

Questa revisione di studi RCT ha analizzato se le CAM possono essere utili per gestire diverse problematiche dei pazienti nefrologici, in particolare per alleviare il prurito, per aumentare la capacità respiratoria, per migliorare la qualità del sonno, per migliorare valori ematochimici, per ridurre il dolore e l'ansia. I primi studi sulle CAM in nefrologia risalgono ai primi anni 2000 ma solo attorno al 2013-2014 sono stati pubblicati numerosi articoli di studi che indagavano in maniera sistematica gli effetti delle CAM. La maggior parte di questi studi sono stati condotti in Cina, Taiwan, Iran, Brasile, Turchia, Paesi dove le CAM sono più diffuse (23). Le CAM sono terapie complementari a quelle della medicina tradizionale. Nei nostri articoli sono state trattate diverse importanti CAM come la medicina tradizionale cinese, le erbe terapeutiche, l'agopuntura, il training respiratorio, il rilassamento Benson, l'aromaterapia, la riflessologia, la musica, lo yoga.

La medicina tradizionale cinese utilizza molte piante medicinali non presenti nei Paesi occidentali, e gli studi mettono in evidenza come l'assunzione da parte dei pazienti di particolari erbe medicinali, possa contribuire a ridurre alcuni valori del sangue e delle urine, come: proteinuria, ematuria, eritrocituria, colesterolo, creatinina, trigliceridi.

Chen et al (19) hanno fatto assumere una vasta gamma di piante officinali denominate Shenqi particle non facilmente reperibili nei Paesi occidentali. L'assunzione di questa tipologia di piante può limitare l'uso di molecole come il prednisone e la ciclofosfamide. Risultati evidenti sono stati raggiunti anche con l'uso di Salacia oblonga e chinensis che hanno ridotto i livelli di creatinina $(11,12)$. L'uso di Abelmoschus manihot (25) contribuisce a ridurre la proteinuria e la pressione arteriosa riducendo l'utilizzo di losartan. Nello studio di Li et al (7) dopo 12 settimane di trattamento ai pazienti che ricevevano Huai Qi Haung si è visto un miglioramento di ematuria.

Nello studio di Wu et al (23) i pazienti che avevano effettuato il trapianto renale e sottoposti a ciclosporina, hanno avuto minori effetti collaterali dovuti a ciclosporina se trattati 
con Berberina. Le Zishen Tongli Jiaonang capsule sono state utilizzate per le infezioni del tratto urinario, l'uso di questa erba medicinale è stato sperimentato in un gruppo di pazienti con infezioni urinarie mostrando un tasso di infezioni nel gruppo in trattamento ridotto $(p=<0,01)(17,25)$. Shema-Didi et al (1) hanno osservato che l'utilizzo del succo di melograno può ridurre la pressione sistolica, i trigliceridi e i livelli di HDL. Qiu et al (9) hanno dimostrato come la Rehmannia possa ridurre la proteinuria. Nello studio di Lemos et al (20), l'uso di oli di semi di lino ha ridotto lo stato infiammatorio, e in modo particolare il BMI, le lipoproteine, il colesterolo ( $p=0,001)$.

L'agopuntura è stata la CAM più studiata. Si tratta di una procedura non invasiva, di origine cinese, per stimolare tramite un massaggio o tramite il posizionamento di un ago, meridiani o punti precisi del corpo umano. Utilizzata in punti del corpo specifici e con tempi definiti è risultata utile per alleviare il prurito e per migliorare il sonno. In particolare è risultata utile per la qualità del sonno in diversi studi $(14,22)$ che I'hanno praticata per un mese in punti del corpo uguali (mani e piedi) e nello studio di Arab et al (30) dove veniva praticata con il pollice nei punti del polso. In questi studi il punteggio totale della PSQI ha dimostrato l'efficacia dell'agopuntura $(p<0,001)$. In un altro studio (13) invece non è risultata statisticamente efficace probabilmente perché praticata in punti specifici per la fatigue (gambe). Nello studio di Wang et al (15), praticata in zone del corpo ben studiate come la zona auriculare, ha indotto un miglioramento della qualità di vita (misurata con il KDQOL-SF) in pazienti con malattia cronica renale $(p=0,001)$.

Che-Yi et al (28) nel 2005 hanno studiato gli effetti dell'agopuntura praticata per un mese nel punto Quchi (LI11) nel gomito ed è emerso che aiuta ad attenuare il prurito uremico con effetti che durano anche per tre mesi dalla fine del trattamento. Questo studio segnala anche un effetto collaterale ovvero il dolore manifestato in tre pazienti il quale però si è risolto spontaneamente dopo un giorno.

Un'altra tipologia di CAM molto studiata in ambito nefrologico è il training respiratorio: esercizi di respirazione guidata effettuati durante l'emodialisi. Sono stati utilizzati per migliorare la capacità respiratoria che tende a diminuire nei pazienti in dialisi. In uno studio (6) gli esercizi venivano praticati per 20 minuti e consistevano in un minuto di respirazione forzata seguito da un minuto di riposo. Al termine dello studio è emerso un miglioramento significativo dei valori della capacità respiratoria. Risultati simili sono comparsi anche in uno studio successivo (8) il quale ha osservato nei pazienti sottoposti al training respiratorio anche il miglioramento della qualità di vita correlata al miglioramento del sonno e alla riduzione del dolore e della fatigue. Infatti gli esercizi di respirazione sono stati studiati anche come rimedio per il sonno disturbato. Nel 2015 Tsai et al (32) hanno sottoposto i pazienti in dialisi a 20 minuti di respirazione guidata. Non ci sono stati risultati significativi per quanto riguarda la qualità del sonno ma ci sono stati dei miglioramenti nella gravità della depressione e nella qualità di vita relativa alla salute. È noto infatti che le patologie renali e le lunghe sedute di dialisi comportano una riduzione della forza muscolare e della capacità respiratoria con conseguenti disturbi nella vita quotidiana: stanchezza, sonno disturbato ecc. che a lungo andare possono portare alla depressione. Gli esercizi respiratori sono in grado di essere d'aiuto al recupero delle forze muscolari e sono anche un possibile rimedio per il sonno e per la depressione $(6,32)$.

II rilassamento Benson è una tecnica studiata da Benson nel 1975 che porta al completo rilassamento di tutti i muscoli. Consiste in sedute di 20 minuti in cui è indicato sedersi in una posizione comoda, chiudere gli occhi, rilassare tutti i muscoli a partire dai piedi, respirare attraverso il naso, prestare attenzione al suono del respiro, e dire la parola "uno" tranquillamente a se stessi quando si espira. Gli effetti di questa tecnica sul dolore e sul sonno in pazienti in emodialisi sono stati analizzati da due studi $(10,29)$ : nel 2013 hanno dimostrato una diminuzione significativa nel punteggio totale della PSQI. Nel 2014 hanno constatato una diminuzione dell'intensità del dolore dopo otto settimane di trattamento.

II sonno disturbato nei pazienti con ESRD ha un impatto negativo sulle componenti fisiche e mentali e porta a una diminuzione delle prestazioni e disfunzioni cognitive e mnemoniche. L'agopuntura, il training respiratorio e il rilassamento Benson possono essere d'aiuto anche per questo disturbo, sia agendo nella fase lieve sia nella fase acuta della manifestazione del problema $(4,6,22,32)$.

L'aromaterapia, uso di oli essenziali derivati da piante, a base di lavanda, è stata studiata da Bagheri-Nesami et al (17) come rimedio al dolore provocato dall'inserimento dell'ago nella fistola. Dopo tre interventi, hanno rilevato attraverso la scala VAS una diminuzione dell'intensità del dolore. L'utilizzo della lavanda però non è risultato efficace come rimedio per la fatigue e di conseguenza per il dolore cronico (28).

Lo yoga, che deriva dalla parola "unire", connota "I'unione della natura umana al superiore" ovvero permette a una persona di modificare le risposte mentali e corporee attraverso esercizi di respirazione (pranayama), di postura (asana), e devozione e meditazione (dhyana). La pratica di esercizi di base di yoga come ritmo lento e coordinato della respirazione, focalizzazione sulle risposte del corpo, può portare a una diminuzione della PSQI e quindi al miglioramento del sonno (4). L'ascolto della musica cattura l'attenzione del paziente distogliendo paura e ansia. La musicoterapia è stata praticata (6) facendo ascoltare musica d'arte turca a pazienti dializzati, al termine hanno misurato una diminuzione dell'ansia.

La musica, come anche l'aromaterapia, lo yoga, il rilassamento Benson, il training respiratorio infatti, hanno un'elevata capacità di creare rilassamento. Relax che porta effetti sia fisi$\mathrm{ci}$, come la distensione dei muscoli, sia effetti psicologici, come il distogliere da ansie e preoccupazioni derivanti dalla patologia e da ciò che essa comporta. Vivere con una patologia renale, la maggior parte delle volte, implica lunghe sedute di 
emodialisi, ospedalizzazioni, diete restrittive, dolore. L'uomo che si deve sottoporre a tutto questo, inevitabilmente incontra momenti di tensione e paure che incidono negativamente sul suo benessere sia psicologico che fisico $(4,5,29,32)$.

Per trattare il prurito, il dolore e l'ansia sono state sperimentate anche altre terapie alternative. Una di queste è la riflessologia, una pratica antica, che consiste nell'applicare una pressione nei punti riflessi nelle mani e nei piedi corrispondenti a ghiandole endocrine di tutto il corpo. La riflessologia è stata praticata durante l'emodialisi nei punti corrispondenti a dove i pazienti provavano dolore, fatigue e crampi oppure prima della seduta di dialisi nei piedi. In entrambi i casi la riflessologia ha migliorato la qualità del sonno e il dolore misurato con la scala VAS è diminuito notevolmente $(21,31)$.

Ci sono stati degli altri studi che hanno provato ad alleviare il prurito con la terapia termale e con l'omeopatia. La terapia termale utilizzata da $\mathrm{Hsu}$ et al (2) consisteva in raggi infrarossi diretti in un punto preciso del piede, i quali accelerano la circolazione del sangue che rigenera i tessuti. Hanno utilizzato per misurare il prurito la forma breve della scala validata McGill Pain Questionnaire e hanno constatato che il prurito diminuisce di molto.

L'omeopatia, la più diffusa tra le medicine non convenzionali, prevede l'utilizzo di sostanze che possono dare benefici simili ai farmaci. Per il prurito è stato studiato l'effetto di alcune sostanze omeopatiche, come la Calcarea phosphorica, e si è constatato una diminuzione dell'intensità del prurito significativa (18). Nello studio di Nakhaee et al (26) l'uso della lozione cutanea a base di Avena sativa non è risultata particolarmente efficace per il prurito probabilmente perché le cause del prurito nei pazienti nefropatici non sono solo cutanee.

Gli studi analizzati riportano i sintomi più diffusi nei pazienti con ESRD, sintomi che oltre a dare discomfort possono impedire il recupero da malattie o anche peggiorare la patologia $(10,22)$.

\section{Conclusioni}

Attraverso questa revisione di studi RCT è emerso che, anche se le evidenze degli studi reperiti sono limitate, le CAM possono essere d'aiuto per il trattamento e il controllo dei problemi che i pazienti nefropatici possono accusare come prurito, ridotta capacità respiratoria e muscolare, scarsa qualità del sonno, valori ematochimici fuori range, dolore, ansia. Le CAM possono aiutare a diminuire l'intensità di questi sintomi ma emerge il fatto che nei Paesi occidentali non vengono prese in considerazione per un'integrazione con la medicina tradizionale. Molti pazienti sostengono di usare costantemente terapie complementari, ma pochi chiedono al medico o all'infermiere, chiedono solo consiglio agli amici o conoscenti (3). Questo è particolarmente pericoloso perché i pazienti con poca capacità di escrezione renale, non tengono conto dei potenziali rischi e della sicurezza della loro salute. Interazioni tra CAM e farmaci possono produrre una aumento della tossicità, cambiamenti della farmacocinetica, con peg- gioramento della funzionalità renale e delle condizioni generali della persona. È auspicabile che il medico e l'infermiere, attraverso una buona comunicazione, si informino sulle CAM che il paziente nefrologico utilizza per evitare potenziali rischi e pericolose interazioni.

\section{Disclosures}

Financial support: No financial support was received for this submission. Conflict of interest: The authors have no conflict of interest.

\section{Bibliografia}

1. Shema-Didi L, Kristal B, Sela S, Geron R, Ore L. Does Pomegranate intake attenuate cardiovascular risk factors in hemodialysis patients? Nutr J. 2014;13-8.

2. Hsu MC, Chen HW, Hwu YJ, Chanc CM, Liu CF. Effects of thermal therapy on uremic pruritus and biochemical parameters in patients having haemodialysis. J Adv Nurs. 2009;65(11):2397-408.

3. Duncan HJ, Pittman S, Govil A, et al. Alternative medicine use in dialysis patients: potential for good and bad! Nephron Clin Pract. 2007;105(3):c108-13.

4. Yurtkuran M, Alp A, Yurtkuran M, Dilek K. A modified yogabased exercise program in hemodialysis patients: a randomized controlled study. Complement Ther Med. 2007;15(3):164-71.

5. Cantekin I, Tan M. The influence of music therapy on perceived stressors and anxiety levels of hemodialysis patients. Ren Fail. 2013;35(1):105-9.

6. Figueiredo RR, Castro AA, Napoleone FM, Faray L, de Paula Júnior AR, Osório RA. Respiratory biofeedback accuracy in chronic renal failure patients: a method comparison. Clin Rehabil. 2012;26(8):724-32.

7. Li LT, Shi MY, Wei SY, Li T, Li B. Huai Qi Huang ameliorates proteinuria and hematuria in mild IgA nephropathy patients: a prospective randomized controlled study. J Formos Med Assoc. 2013;112(12):766-72.

8. Pellizzaro CO, Thomé FS, Veronese FV. Effect of peripheral and respiratory muscle training on the functional capacity of hemodialysis patients. Ren Fail. 2013;35(2):189-97.

9. Qiu H, Fan W, Fu P, et al. General acteoside of Rehmanniae leaves in the treatment of primary chronic glomerulonephritis: a randomized controlled trial. Afr J Tradit Complement Altern Med. 2013;10(4):109-15

10. Rambod M, Pourali-Mohammadi N, Pasyar N, Rafii F, Sharif F. The effect of Benson's relaxation technique on the quality of sleep of Iranian hemodialysis patients: a randomized trial. Complement Ther Med. 2013;21(6):577-84.

11. Singh RG, Rathore SS, Kumar R; Usha, Agarwal A, Dubey GP. Nephroprotective role of salacia chinensis in diabetic CKD patients: a pilot study. Indian J Med Sci. 2010;64(8):378-84.

12. Singh RG, Rathore SS, Wani IA; Usha, Agrawal A, Dubey GP. Effects of Salacia oblonga on cardiovascular risk factors in chronic kidney disease patients: a prospective study. Saudi J Kidney Dis Transpl. 2015;26(1):61-6.

13. Tsay SL. Acupressure and fatigue in patients with end-stage renal disease-a randomized controlled trial. Int J Nurs Stud. 2004;41(1):99-106.

14. Tsay SL, Chen ML. Acupressure and quality of sleep in patients with end-stage renal disease--a randomized controlled trial. Int J Nurs Stud. 2003;40(1):1-7.

15. Wang S, Chen Z, Fu P, et al. Use of auricular acupressure to improve the quality of life in diabetic patients with chronic kidney diseases: a prospective randomized controlled trial. 
Evid Based Complement Alternat Med. 2014;2014:343608.

16. Zhang $M$, Zhang $D, X u Y$, Duo $X$, Zhang $W$. A clinical study on the treatment of urinary infection with Zishen Tongli Jiaonang. J Tradit Chin Med. 2005;25(3):182-5.

17. Bagheri-Nesami M, Espahbodi F, Nikkhah A, Shorofi SA, Charati JY. The effects of lavender aromatherapy on pain following needle insertion into a fistula in hemodialysis patients. Complement Ther Clin Pract. 2014;20(1):1-4.

18. Cavalcanti AM, Rocha LM, Carillo R Jr, Lima LU, Lugon JR. Effects of homeopathic treatment on pruritus of haemodialysis patients: a randomised placebo-controlled double-blind trial. Homeopathy. 2003;92(4):177-81.

19. Chen Y, Deng Y, Ni Z, et al. Efficacy and safety of traditional chinese medicine (Shenqi particle) for patients with idiopathic membranous nephropathy: a multicenter randomized controlled clinical trial. Am J Kidney Dis. 2013;62(6):1068-76.

20. Lemos JR, Alencastro MG, Konrath AV, Cargnin M, Manfro RC. Flaxseed oil supplementation decreases C-reactive protein levels in chronic hemodialysis patients. Nutr Res. 2012;32(12):921-7.

21. Ozdemir G, Ovayolu N, Ovayolu O. The effect of reflexology applied on haemodialysis patients with fatigue, pain and cramps. Int J Nurs Pract. 2013;19(3):265-73.

22. Shariati A, Jahani $S$, Hooshmand $M$, Khalili $N$. The effect of acupressure on sleep quality in hemodialysis patients. Complement Ther Med. 2012;20(6):417-23.

23. Wu X, Li Q, Xin H, Yu A, Zhong M. Effects of berberine on the blood concentration of cyclosporin $A$ in renal transplanted recipients: clinical and pharmacokinetic study. Eur J Clin Pharmacol. 2005;61(8):567-72.

24. Zhang M, Zhang D, Zhang W, Liu S, Zhang M. Treatment of chronic renal failure by supplementing the kidney and invigorating blood flow. J Tradit Chin Med. 2004;24(4):247-51.
25. Zhang L, Li P, Xing CY, et al. Efficacy and safety of Abelmoschus manihot for primary glomerular disease: a prospective, multicenter randomized controlled clinical trial. Am J Kidney Dis. 2014;64(1):57-65.

26. Nakhaee S, Nasiri A, Waghei Y, Morshedi J. Comparison of Avena sativa, vinegar, and hydroxyzine for uremic pruritus of hemodialysis patients: a crossover randomized clinical trial. Iran J Kidney Dis. 2015;9(4):316-22.

27. Che-Yi C, Wen CY, Min-Tsung K, Chiu-Ching H. Acupuncture in haemodialysis patients at the Quchi (LI11) acupoint for refractory uraemic pruritus. Nephrol Dial Transplant. 2005;20(9):1912-5.

28. Bagheri-Nesami M, Shorofi SA, Nikkhah A, Espahbodi F, Ghaderi Koolaee FS. The effects of aromatherapy with lavender essential oil on fatigue levels in haemodialysis patients: A randomized clinical trial. Complement Ther Clin Pract. 2016;22:33-7.

29. Rambod M, Sharif F, Pourali-Mohammadi N, Pasyar N, Rafii F. Evaluation of the effect of Benson's relaxation technique on pain and quality of life of haemodialysis patients: a randomized controlled trial. Int J Nurs Stud. 2014;51(7):964-73.

30. Arab Z, Shariati AR, Asayesh H, Vakili MA, Bahrami-Taghanaki $\mathrm{H}$, Azizi $\mathrm{H}$. A sham-controlled trial of acupressure on the quality of sleep and life in haemodialysis patients. Acupunct Med. 2016;34(1):2-6.

31. Unal KS, Balci Akpinar R. The effect of foot reflexology and back massage on hemodialysis patients' fatigue and sleep quality. Complement Ther Clin Pract. 2016;24:139-44.

32. Tsai SH, Wang MY, Miao NF, Chian PC, Chen TH, Tsai PS. CE: original research: The efficacy of a nurse-led breathing training program in reducing depressive symptoms in patients on hemodialysis: a randomized controlled trial. Am J Nurs. 2015;115(4):24-32. 